\title{
Synthesis, Growth and Characterization of Sodium Mixed Cadmium Oxalate Crystals
}

\author{
F. Daisy Selasteen ${ }^{*}$, S. Alfred Cecil Raj ${ }^{2}$, A. Alagappa Moses ${ }^{3}$, F. Emalda Prince ${ }^{1}$, \\ R. Esther Getsy ${ }^{1}$, R. Elakkiya ${ }^{1}$ \\ ${ }^{1}$ Department of Physics, Bishop Heber College, Tiruchirappalli, India \\ ${ }^{2}$ Department of Physics, St. Joseph's College, Tiruchirappalli, India \\ ${ }^{3}$ Department of Environmental Sciences, Bishop Heber College, Tiruchirappalli, India \\ Email: ${ }^{*}$ fdaisyallen@yahoo.com
}

Received 6 February 2016; accepted 11 April 2016; published 14 April 2016

Copyright (C) 2016 by authors and Scientific Research Publishing Inc.

This work is licensed under the Creative Commons Attribution International License (CC BY). http://creativecommons.org/licenses/by/4.0/

(c) (i) Open Access

\begin{abstract}
The newly synthesized $\mathrm{CdNa}_{2} \cdot 2 \mathrm{C}_{2} \mathrm{O}_{4} \cdot 2 \mathrm{H}_{2} \mathrm{O}$ crystals having a size of $0.30 \times 0.25 \times 0.20 \mathrm{~mm}^{3}$ were grown by the single-diffusion reaction method in the silica gel medium at $28^{\circ} \mathrm{C}$ temperature. The crystalline nature of crystals was determined in 20 days by 1) varying the concentrations of the feed solutions and the gel $\mathrm{pH}$ level, 2) changing the specific gravity of the feed solution, and 3) changing the gel aging period. The crystals were characterized by using the methods such as powder X-ray diffraction, FTIR, UV, SEM-EDAX, TGA/DTA, and micro-hardness test. The structure of as-grown crystals was determined by the powder crystal XRD patterns. FTIR \& SEM-EDAX spectral analyses were used to confirm the presence of the required functional groups of as grown crystals. UV absorption spectrum, the absorption peaks at 224.64 nanometer, determined the energy gap. TGA/DTA thermo gram was used to detect the thermal stability of sodium mixed cadmium oxalate dihydrate crystals. The present grown crystals were brittle and also plastic in nature was identified by Vickers micro-hardness test.
\end{abstract}

\section{Keywords}

$\mathrm{CdNa}_{2} \cdot 2 \mathrm{C}_{2} \mathrm{O}_{4} \cdot 2 \mathrm{H}_{2} \mathrm{O}$ Crystal Growth, XRD, FTIR, UV, TGA/DTA, SEM-EDAX, Micro Hardness

\section{Introduction}

Single oxalate crystals can be prepared by using the gel growth single-diffusion technique. Silica gel method of single diffusion reaction technique is capable of yielding crystals of high optical perfection and wide morphology and is quite suitable for the growth of well-developed and notably transparent crystals of pure metal oxalates

${ }^{*}$ Corresponding author.

How to cite this paper: Selasteen, F.D., Raj, S.A.C., Moses, A.A., Prince, F.E., Getsy, R.E. and Elakkiya, R. (2016) Synthesis, Growth and Characterization of Sodium Mixed Cadmium Oxalate Crystals. Journal of Crystallization Process and Technology, 6, 11-20. http://dx.doi.org/10.4236/icpt.2016.62002 
of cadmium oxalate, barium oxalate, strontium oxalate, calcium oxalate, and zirconium oxalate. The TGA/DTA, dielectric and optical characteristics of cadmium and sodium oxalates explained their band gap energy and thermal stability found applications in optoelectronic industries and in the similar way like barium oxalate, strontium oxalate and zirconium oxalate have been reported because of their potential applications in pyrotechnology and opto electronic industries [1]-[5]. Among the metallic ions studied, $\mathrm{Cd}$ and Na ions have attracted a great deal of interest because it has rather simple chemistry and coordination geometry. Henceforth, we have taken an attempt for the first time to grow sodium mixed cadmium dioxalate dehydrate (SCO) crystals in silica gel medium using single diffusion reaction technique. It acts as bidentate, donor ligand and forms with metal centers chains. Combined with the presence of water molecules directly linked to the metal ions or weakly bonded to the frame work, this leads to a very large variety of structural architectures in three dimensions. In the field of industrial crystallization and semiconductor industries, much importance is given to the addition of foreign particles with the pure crystals in crystallization process. The incorporation of impurities to these crystals is responsible for the specific changes of their physical properties [6]. A known property of oxalates is their insolubility in water, which is useful in separating the transition elements from the mineral as oxalates precipitate [7]. Therefore, to obtain good quality of single crystals of oxalates, no evaporation and high-temperature methods can be used to grow oxalates as they decompose before melting [8]. But the insolubility of oxalates in water [9] is an advantage in growing these crystals by silica gel method, and also, the gel grown transition metal oxalate materials have attracted many solid state physicists to study their thermal properties and their potential applications in the field of organic semiconductors, analytical chemistry, and in industries [10]. However, the principal aim of the present study is mainly focused to study the 1) growth of sodium mixed cadmium dioxalate (SCO) crystals in single diffusion technique, 2) optical properties, 3) changes in the physicochemical properties as a function of increasing temperature with time, and 4) mechanical properties of as grown SCO crystals and identifies their potential functional significances in the electronic industries.

\section{Experimental}

\subsection{Preparation of Single Crystals}

The growth of sodium-mixed cadmium oxalate crystals was carried out in silica gel media by adopting the single-diffusion technique. The high-purity elements such as cadmium chloride, sodium chloride, oxalic acid, and sodium meta silicate with AR grade were used as the starting materials in the single-diffusion method at a temperature of $28^{\circ} \mathrm{C}$.

\subsection{Crystal Growth of $\mathrm{CdNa}_{2} \cdot 2(\mathrm{COO})_{2} \cdot 2 \mathrm{H}_{2} \mathrm{O}$}

The optimized growth of gel grown crystals is accomplished by controlled diffusion process. This control of nucleation growth is greatly influenced by single diffusion reaction technique. A test tube (height, $15 \mathrm{~cm}$; diameter, $1.5 \mathrm{~cm}$ ) was selected as a container for growing the crystals in single-diffusion method. Silica gel was prepared by adding sodium meta silicate solution with a specific gravity of 1.03 to oxalic acid $(1 \mathrm{M})$, drop by drop with continuous stirring to avoid excessive local ion concentration which may cause premature local gelling and make the final solution inhomogeneous. This solution was set with a desired $\mathrm{pH}$ range of 4.5 and poured into different test tubes for setting the gel. The gel setting period was exactly 6 days and then the supernatant solution (cadmium chloride $(1 \mathrm{M})$ ) was poured over the set gel with the help of a pipette in order to avoid the surface damage and breakage of the gel in the test tube. To increase the crystal size, $10 \mathrm{ml}$ of $4 \mathrm{M} \mathrm{NaCl}$ was added with the $1 \mathrm{M}$ of cadmium chloride solution on the set gel to each test tube. The expected and the desired quality of crystals larger in size, transparent, and prismatic shape were grown in the gel within in the period of 15 - 20 days. Typical growth conditions and photographs of as-grown crystals are displayed in Table 1 and Figure 1(a) and Figure 1(b).

When sodium meta silicate goes into a solution, mono silicic acid is expected to be produced [11] according to the following reaction.

$$
\mathrm{Na}_{2} \mathrm{SiO}_{3}+3 \mathrm{H}_{2} \mathrm{O} \rightarrow \mathrm{H}_{4} \mathrm{SiO}_{4}+2 \mathrm{NaOHR} .
$$

This sodium hydroxide is expected to react with oxalic acid, diffusing in a gel from the supernatant solution and forming sodium mixed cadmium oxalate crystals (SCO) by the following reaction. 
Table 1. Growth conditions of $\mathrm{CdNa}_{2} \cdot 2 \mathrm{C}_{2} \mathrm{O}_{4} \cdot 2 \mathrm{H}_{2} \mathrm{O}$ single crystals.

\begin{tabular}{cc}
\hline Condition & Single diffusion \\
\hline Density of sodium meta silicate & 1.03 \\
$\mathrm{pH}$ of gel & 4.5 \\
Concentration of $\mathrm{CdCl}_{2}$ & $1 \mathrm{M}$ \\
Gel setting period & 6 days \\
Gel aging & 48 hours \\
Period of growth & 20 days \\
Temperature & $28^{\circ} \mathrm{C}$ \\
Quality & Transparent \\
Size & $0.30 \times 0.25 \times 0.20 \mathrm{~mm}^{3}$ \\
\hline
\end{tabular}

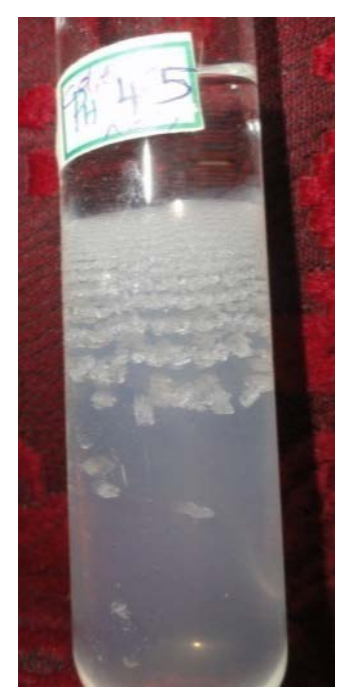

(a)

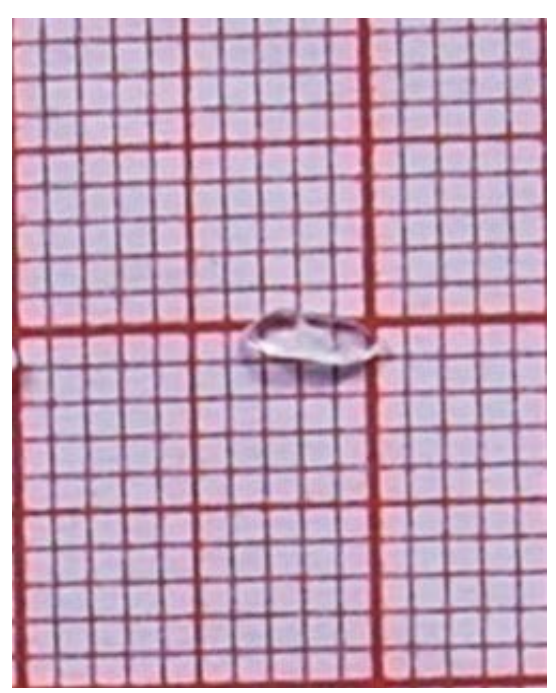

(b)

Figure 1. Growth of $\mathrm{CdNa}_{2} \cdot 2 \mathrm{C}_{2} \mathrm{O}_{4} \cdot 2 \mathrm{H}_{2} \mathrm{O}$ crystals: (a) $\mathrm{CdNa}_{2} \cdot 2 \mathrm{C}_{2} \mathrm{O}_{4} \cdot 2 \mathrm{H}_{2} \mathrm{O}$ crystals formation in silica gel; (b) Photograph of as-grown $\mathrm{CdNa}_{2} \cdot 2 \mathrm{C}_{2} \mathrm{O}_{4} \cdot 2 \mathrm{H}_{2} \mathrm{O}$ crystal with a size of $0.30 \times 0.25 \times 0.20 \mathrm{~mm}^{3}$.

$$
2 \mathrm{NaOH}+2 \mathrm{H}_{2} \mathrm{C}_{2} \mathrm{O}_{4}+\mathrm{CdCl}_{2} \rightarrow \mathrm{CdNa}_{2} 2 \mathrm{C}_{2} \mathrm{O}_{4} \cdot 2 \mathrm{H}_{2} \mathrm{O}+2 \mathrm{HCl} \cdot \mathrm{R}
$$

\section{Characterization}

The powder XRD pattern of as grown SCO crystals were carried out by powder XPERT-PRO X-ray diffract meter using the intense X-ray of wavelength $1.5406 \AA(\mathrm{Cu} \mathrm{K} \alpha)$ at a scan speed of $1 \%$ minute. FTIR absorption spectrum of as grown SCO crystal was recorded using RX1 model, Perkin Elmer (make) FTIR spectrometer in the region $400-4000 \mathrm{~cm}^{-1}$. SEM-EDAX spectrum of as grown SCO crystals were analyzed using VEGA3 TESCAN at the scanning image range is $2.73 \mathrm{kx}$ to analyze the observation defects. TGA and DTA of grown crystals were carried out using the Mettler TA4000 system in the argon gas atmosphere to study the thermal stability and the decomposition stages of as grown SCO crystals. UV-VIS-NIR Absorption spectrum was recorded in the Lambda 35 instrument with a scanning speed of $480 \mathrm{~nm} /$ min between the wave length ranges of $190 \mathrm{~nm}$ and $1100 \mathrm{~nm}$ to determine the band gap energy. The instrument of Vickers Micro hardness tester attached to a metallurgical microscope, the video measuring systems with offset of diagonal tips of indenter is $<25 \mathrm{~mm}$ and the load resolution $0.001 \mathrm{~N}$ are used to study the hardness numeral of sodium mixed cadmium oxalate crystal at $32^{\circ} \mathrm{C}$ to calculate the mechanical strength of the title compound. 


\section{Results and Discussion}

\subsection{XRD Analysis}

The powder XRD pattern of the sample is shown in Figure 2. The well defined peaks at specific $2 \theta$ values show high crystalline nature of the grown crystals. The observed XRD pattern of this work was indexed using the XRDML software package. Obtained d-spacing and the Miller indices (hkl) are in agreement with the standard values (JCPDS Card No.53-0085) reported. Hence it is likely that the anhydrate crystal has similar crystal system with the standard crystal data which is triclinic that have been already reported [12].

\subsection{Functional Group Identification of $\mathrm{CdNa}_{2} \cdot 2 \mathrm{C}_{2} \mathrm{O}_{4} \cdot 2 \mathrm{H}_{2} \mathrm{O}$ Crystal Using FTIR Spectrum}

FTIR absorption spectrum of as grown SCO crystals shown in Figure 3. The cadmium molecule is the most basic, and therefore, the presence of oxalate groups is revealed in the FTIR spectrum. An intense band of strong

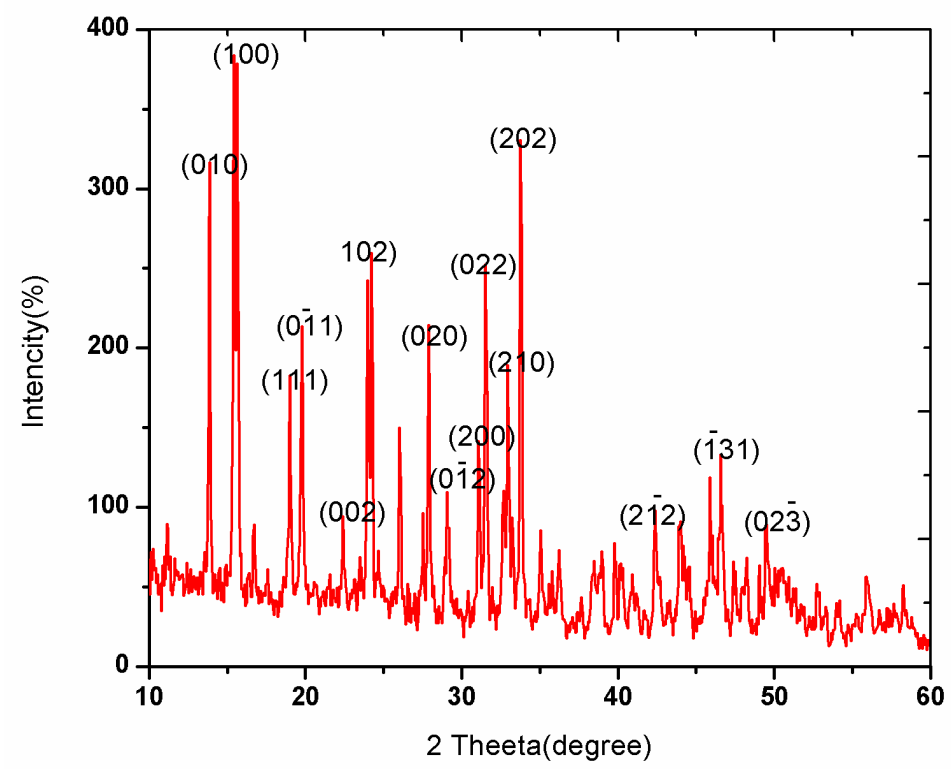

Figure 2. The powder XRD pattern of as-grown $\mathrm{CdNa}_{2} \cdot 2 \mathrm{C}_{2} \mathrm{O}_{4} \cdot 2 \mathrm{H}_{2} \mathrm{O}$ crystal.

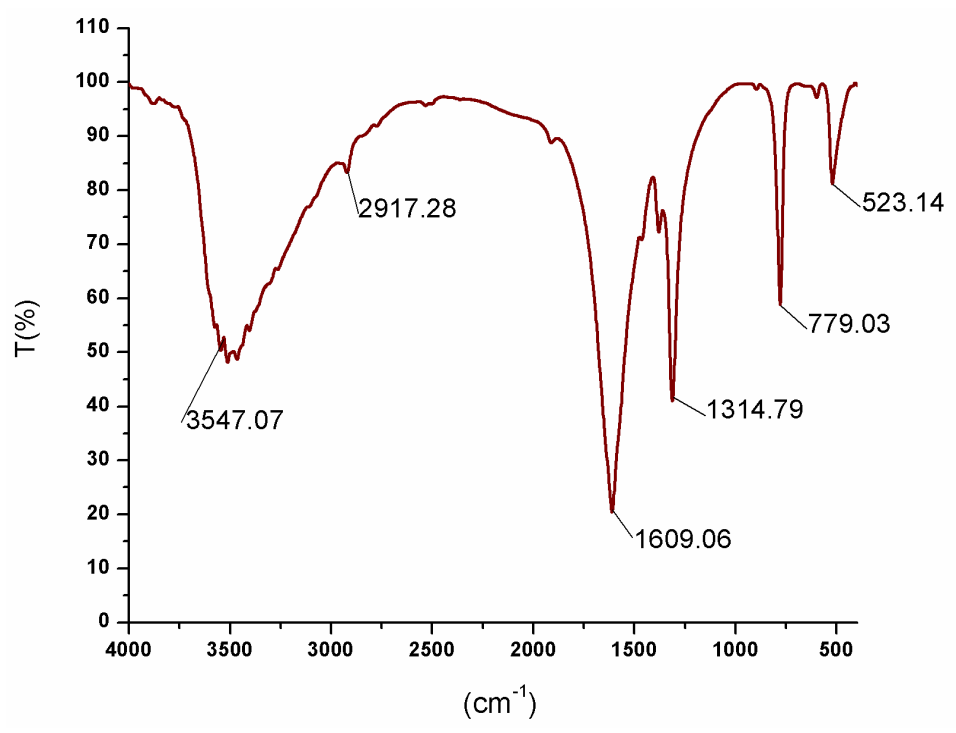

Figure 3. FTIR spectrum of the grown $\mathrm{CdNa}_{2} \cdot 2 \mathrm{C}_{2} \mathrm{O}_{4} \cdot 2 \mathrm{H}_{2} \mathrm{O}$ crystals. 
absorption around $3547.07 \mathrm{~cm}^{-1}$ and protonated by the carboxyl group (COOH) gives hydrogen bonding [13], which is attributed to O-H stretching vibration for the confirmative group of water on hydration. The broadness occurring in absorption bands appears at $1609.06 \mathrm{~cm}^{-1}$, which is supported by the presence of oxalate ion by $\mathrm{O}-\mathrm{H}$ bending vibration [14]. Regions below $1650 \mathrm{~cm}^{-1}$ are referred as the fingerprint for many organic compounds, and also, the well-pronounced sharp peak at $1314.79 \mathrm{~cm}^{-1}$ represents the $\mathrm{CO}_{2}$ symmetric stretching. The very sharp peak that lies at $779.03 \mathrm{~cm}^{-1}$ and $523.14 \mathrm{~cm}^{-1}$ shows the metal-oxygen bonds. These wave numbers are compared with the pure cadmium oxalate crystals [15] and it was found that they were fairly dependent on the band assignments of required functional groups are shown in Table 2.

\subsection{SEM-EDAX Measurements}

He increasing demand for crystals of better perfection for use in the fabrication of electronic devices and in the understanding of the plastic deformation leads to analyze the defects in crystal. The SEM micrograph is shown in Figure 4(a). The micro morphology is photographed and analyzed under the optical microscope. The surfaces are rectangle in shape and are aligned along the flat surface. The rectangle pattern of the flat surface contains few rock and valley shaped dislocations due to the high kink nucleation. The kink nucleation is principally controlled by the successful under saturation of the supernatant solutions. The dislocations may also be due to plastic deformation caused by thermal stresses. Thus the results of SEM are consistent with XRD analysis. The changes in the EDAX measurements are made at different points on the surface of crystals are given in Figure 4(b) and the presence of required atoms of average weight $\&$ atomic percentage values obtained is shown in Table 3. The spectrum confirms the presence of expected major elements like cadmium, sodium, carbon and oxygen of the title compound.

Table 2. FTIR spectrum for functional groups identification of pure $\mathrm{CdC}_{2} \mathrm{O}_{4} \cdot 3 \mathrm{H}_{2} \mathrm{O}$ versus $\mathrm{CdNa}_{2} \cdot 2 \mathrm{C}_{2} \mathrm{O}_{4} \cdot 2 \mathrm{H}_{2} \mathrm{O}$ crystals.

\begin{tabular}{ccc}
\hline Wave number of $\mathrm{Cd} \mathrm{C}_{2} \mathrm{O}_{4} \cdot 3 \mathrm{H}_{2} \mathrm{O}\left(\mathrm{cm}^{-1}\right)$ & Wave number of $\mathrm{CdNa}_{2} \cdot 2 \mathrm{C}_{2} \mathrm{O}_{4} \cdot 2 \mathrm{H}_{2} \mathrm{O}\left(\mathrm{cm}^{-1}\right)$ & Band assignment \\
\hline 1600.4 & 1609.06 & C-O with C-C \\
1322.5 & 1314.79 & $\mathrm{C}=\mathrm{O}$ \\
$776 \& 521.8$ & $779.03 \& 523.14$ & M-O \\
$3586.8,3106.7 \& 2919.3$ & $3547.07 \& 2917.8$ & $\mathrm{O}-\mathrm{H}$ \\
\hline
\end{tabular}

Table 3. Functional groups of SCO crystal.

\begin{tabular}{cccc}
\hline Element & Weight \% & Atomic \% \\
\hline $\mathrm{C}$ & 66.47 & 23.38 \\
$\mathrm{O}$ & 28.14 & 0.01 \\
$\mathrm{Na}$ & 0.02 & 0.65 \\
$\mathrm{Cd}$ & 5.37 & 100.00 & 0.05 \\
Total &
\end{tabular}

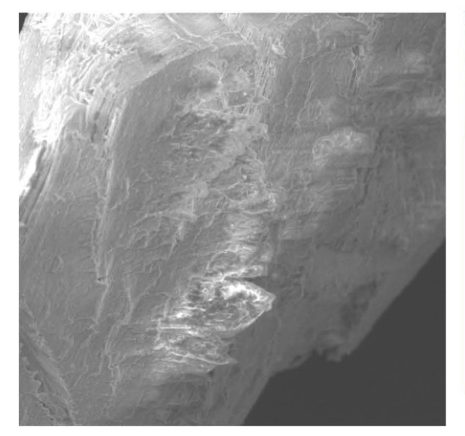

(a)

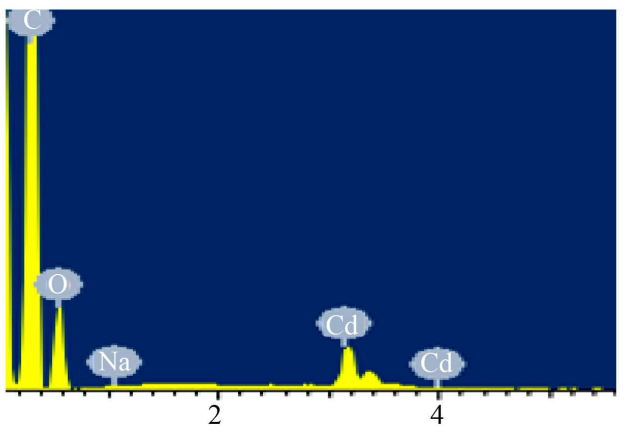

(b)

Figure 4. (a) Morphology of SCO crystals; (b) EDAX spectrum of SCO crystals. 


\subsection{TGA/DTA Thermal analysis}

On the DTA curve, there is one endothermic peak at $135.2^{\circ} \mathrm{C}$ due to the decomposition of sodium mixed cadmium oxalate dihydrate into anhydrous sodium mixed cadmium oxalate. The maximum reaction rate temperatures of $126.4^{\circ} \mathrm{C}$ and $374.4^{\circ} \mathrm{C}$ are denoted by $T_{M}$, resulting in the maximum decomposition states of the compound. The exothermic peak at $400.5^{\circ} \mathrm{C}$ confirms the decomposition of sodium mixed cadmium oxalate into sodium mixed cadmium carbonate and sodium mixed cadmium oxide. In the differential thermal analysis, temperature changes in the sample are due to the reactions caused by phase changes, decomposition, oxidation, reduction, or other chemical reactions [16]. The study of thermal analysis is significant for knowing the different phases and stages of the stability of the compound. TGA thermo-gram from Figure 5 shows two distinct steps. The first step of dehydration starts at $64.8^{\circ} \mathrm{C}$ and ends at $188.18^{\circ} \mathrm{C}$, which shows the formation of anhydrous sodium mixed cadmium oxalate crystal from sodium mixed cadmium oxalate dehydrate crystal, resulting in the weight loss of $20.21 \%$. The second step represents the decomposition of sodium mixed cadmium oxalate crystal into sodium mixed cadmium oxide in the temperature range of $263.02^{\circ} \mathrm{C}$ and $485.8^{\circ} \mathrm{C}$ with $36.6 \%$ weight loss, which shows the release of $\mathrm{CO}_{2}$ and $\mathrm{CO}$ molecules as gases. This variation in the $1^{\text {st }}$ and $2^{\text {nd }}$ stages of mass losses explained that the process is no spontaneous. Fresh calibration of the instrument with minimal ash content $(<3 \%)$ outcomes the TGA values below zero and also the residual mass are negative. This thermal behavior is compared with the already-reported results of the pure cadmium oxalate crystals [17] and found that the stability of as grown crystals is higher than the pure cadmium oxalate crystals and the physiochemical properties of SCO crystals prevent their thermal decomposition during processing.

\subsection{UV Absorption Spectrum}

UV-VIS spectrum studies on pure cadmium oxalate crystals were exhibited both direct and indirect transitions to the band gap energy values: $E_{g}$ (direct) $=4.4 \mathrm{eV}$ and $E_{g}$ (indirect) $=4.2 \mathrm{eV}$ which have already been reported [18]. The recorded optical spectrum of the title compound is found to be active in the UV region having a significant absorption in the lowest cut off wavelength of $224.64 \mathrm{~nm}$ is shown in Figure 6. In the high photon energy region, the energy dependence of absorption coefficient

$$
\alpha=\frac{2.303}{t} \log \left(\frac{1}{T}\right)
$$

where $A$ is the absorbance and $t$ is the thickness of the crystal sample. Absorption coefficient $\alpha$ suggests the

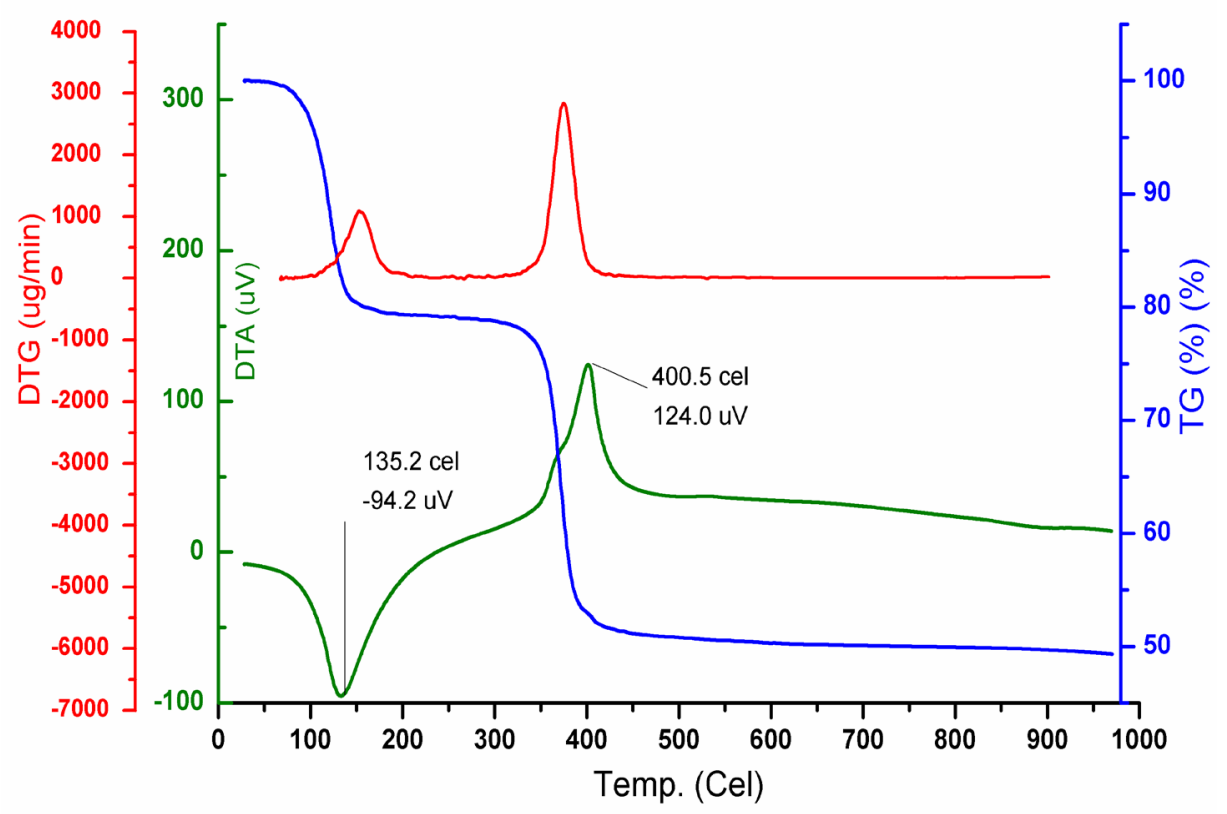

Figure 5. TGA/DTA thermogram of as-grown $\mathrm{CdNa}_{2} \cdot 2 \mathrm{C}_{2} \mathrm{O}_{4} \cdot 2 \mathrm{H}_{2} \mathrm{O}$ crystals. 


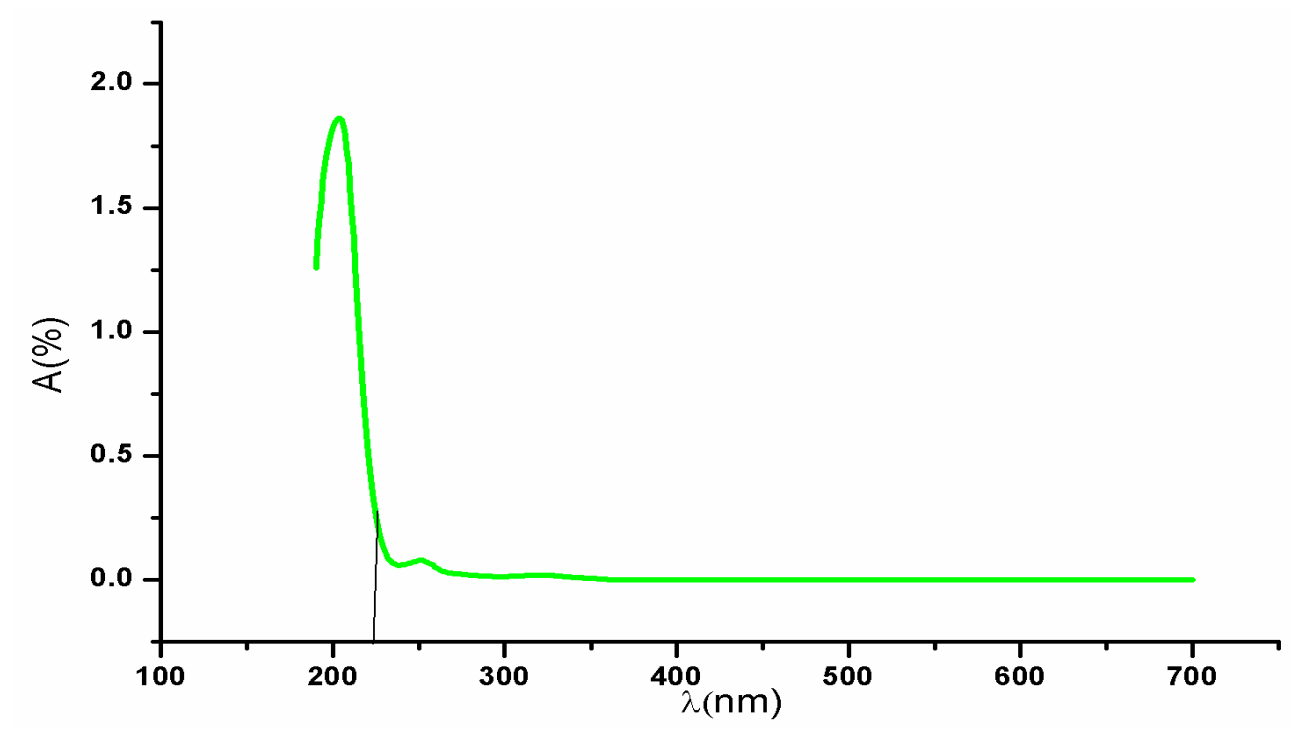

Figure 6. UV-Vis-NIR Absorption spectrum of sodium mixed cadmium oxalate crystal.

occurrence of direct band gap of the crystal obeying the following equation for high photon energies (hv) [19],

$$
(\alpha h v)^{2}=A\left(E_{g}-h v\right)
$$

where " $\alpha$ " is the absorption coefficient, " $h$ " is the Plank's constant, " $A$ " is a constant " $v$ " is the frequency of the incident photon and “ $E_{g}$ ” is the optical band gap. The Tauc's graph [20] plot between $(\alpha h v)^{2}$ and the photon energy $(h v)$ is shown in Figure 7. The extrapolation of the linear part of the graph gives the optical band gap energy value to be $5.5 \mathrm{eV}$ and this wide band gap of SCO crystal confirms the semiconducting activity of as grown-crystal in electronic industries.

\subsection{Micro Hardness Studies}

The study on the hardness numeral of sodium mixed cadmium oxalate crystal is calculated using the relation

$$
H_{v}=1.8544 \cdot \frac{p}{d^{2}} \mathrm{~kg} / \mathrm{mm}^{2}
$$

where $P$ is the applied load in $\mathrm{kg}$ and $d$ is the average diagonal length of the indented impression in millimeter.

Obtained $H_{v}$ for the change in applied loads of the SCO crystals is tabulated in the Table 4. The Hardness number of CO crystals are non linear with the applied test load from the Figure 8 indicates that they are brittle materials. The results of the study are in good agreement with the earlier reported work on the gel grown cadmium oxalate crystals [21].

The slope is taken from the Figure 9 and the index number is found to be

$$
\begin{aligned}
& n=\frac{\mathrm{d} y}{\mathrm{~d} x}=\frac{0.29}{0.14}=2.0714 \\
& \text { Yield strength } \sigma_{v}=\frac{H_{v}}{3} 0.1\left(n^{\prime}\right)^{-2}
\end{aligned}
$$

where $n^{\prime}=n+2$. The average yield strength for various loads is given by $\sigma_{v}=0.0504$. The variation on micro hardness with loads is typically non-linear indicates that the Meyer's law is suitable for the determination of load-dependent hardness.

\section{Conclusion}

FTIR \& EDAX spectral studies confirm the presence of expected functional and metal-oxygen bonded groups of as-grown crystal. Powder XRD reveals the crystalline properties of as-grown crystal. The peaks obtained identify 


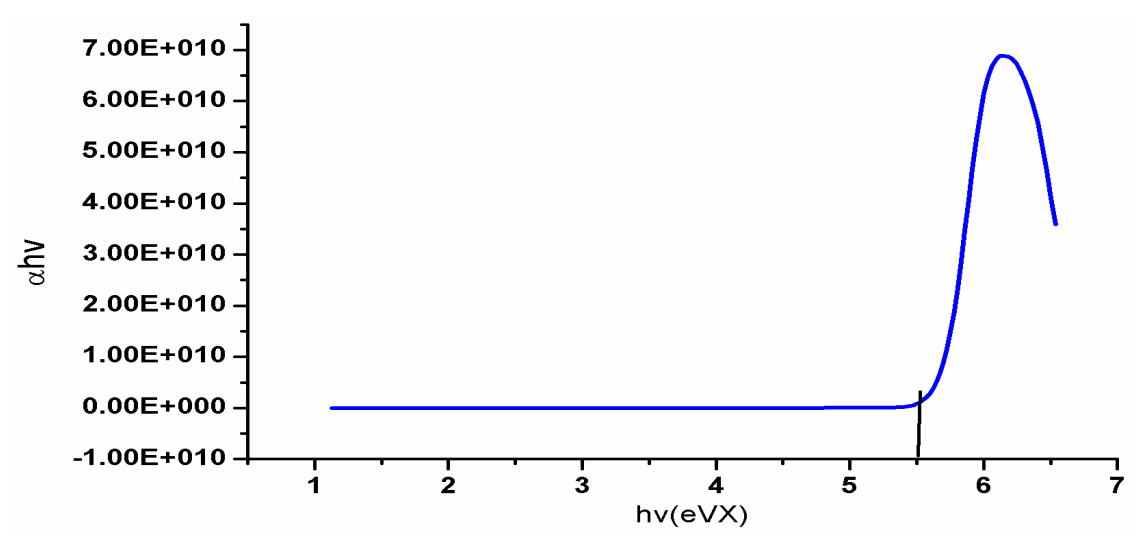

Figure 7. $h v$ vs $(\alpha h v)^{2}$ of sodium mixed cadmium oxalate crystal.

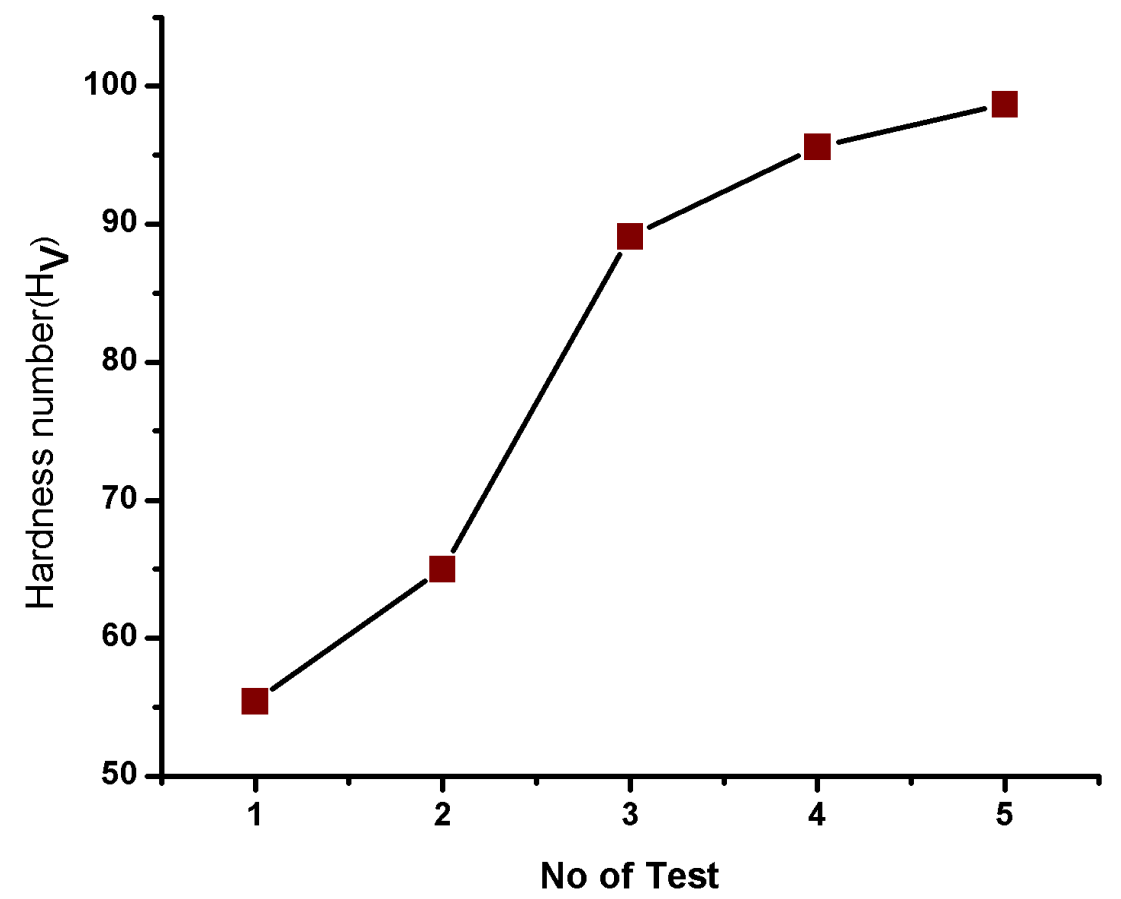

Figure 8 . Hardness $\left(H_{v}\right)$ vs. no. of test $(P)$ of as-grown SCO crystal.

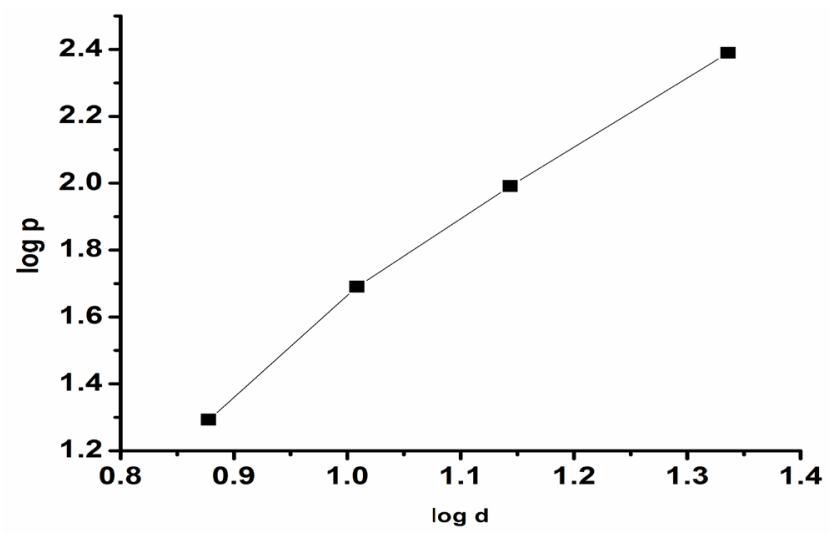

Figure 9. $\log P$ vs. $\log d$ of SCO crystals. 
Table $4 . H_{v}$ value of SCO crystals.

\begin{tabular}{ccccc}
\hline$P(\mathrm{Nm})$ & $D$ (length) & $\log p$ & $\log d$ & $H_{v}$ \\
\hline 9.807 & 5.79 & 0.99 & 0.7626 & 55.4 \\
19.61 & 7.55 & 1.2924 & 0.8779 & 65.0 \\
49.03 & 10.20 & 1.6904 & 1.0086 & 89.1 \\
98.07 & 13.93 & 1.9915 & 1.1439 & 95.6 \\
245.2 & 21.8 & 2.3895 & 1.3360 & 98.7 \\
\hline
\end{tabular}

the grown crystals belonging to triclinic system. Vickers's micro hardness test shows that the variation in $H_{v}$ with a load on the surfaces of sodium mixed cadmium oxalate crystals is typically non-linear and reveals that they are brittle materials. The SEM micrograph shows the good perfection and few defects on the surface of the SCO crystals. The band gap energy, wide transparent nature together with non linearity from UV and the perfect layered linear alignment of the surface studies indicate that the SCO crystals are suitable for the fabrication of materials for opto-electronic devices. TGA thermo gram plots reveal that the present sample is stable at $64.8^{\circ} \mathrm{C}$ supporting its application in the electronic industries and prove that the metal dicarboxilates are the appropriate stabilizing material of PVC to control its thermal decomposition during processing.

\section{Acknowledgements}

The authors are also thankful to University Grants Commission, Grant no.F.NO:4-4/2014-15 (MRPSEM/UGCSERO) for providing financial support under Minor Research Project scheme. We extend our heartfelt thanks to Dr. Justin, Assistant professor, Department of Physics (NIT), Tiruchirappalli and also Mr. Mohan, Sophisticated Analytical Instrument Facilities (SAIF), IIT-Chennai, for providing characterization facilities.

\section{References}

[1] Chelet-Araba, B., Nowogrockia, G., Abrahama, F. and Grandjean, S. (2007) New Alkaline Earth-Zirconium Oxalate $\mathrm{M}_{2} \mathrm{Zr}\left(\mathrm{C}_{2} \mathrm{O}_{4}\right)_{4} \cdot \mathrm{nH}_{2} \mathrm{O}(\mathrm{M}=\mathrm{Ba}$, Sr, Ca) Synthesis, Crystal Structure and Thermal Behavior. Journal of Solid State Chemistry, 177, 4269-4281. http://dx.doi.org/10.1016/j.jssc.2004.06.053

[2] Shedam, M.R. and Venkateswara Rao, A. (1998) Effect of Temperature on Nucleation and Growth of Cadmium Oxalate Single Crystals in Silica Gel. Materials Chemistry and Physics, 52, 263. http://dx.doi.org/10.1016/S0254-0584(97)02042-7

[3] Yu, J.G., Tang, H. and Cheng, B. (2005) Influence of PSSS Additive and Temperature on Morphology and Phase Structure of Calcium Oxalate. Journal of Colloid and Interface Science, 288, 407. http://dx.doi.org/10.1016/j.jcis.2005.03.001

[4] Dalal, P.V., Saraf, K.B., Shimpi, N.G. and Shah, N.R. (2012) Pyro and Kinetic Studies of Barium Oxalate Crystals Grown in Silica Gel. Journal of Crystallization Process and Technology, 2, 156-160. http://dx.doi.org/10.4236/jcpt.2012.24023

[5] Bacce, E.D., Pires, A.M., Davaios, M.R. and Jafelicci Jr., M. (2001) Thermal Decomposition and Rehydration of Strontium Oxalate: Morphological Evoluation. International Journal of Inorganic Materials, 3, 443. http://dx.doi.org/10.1016/S1466-6049(01)00047-2

[6] Jagannathan, N. and Mohan Rao, P. (1993) Studies on Impurity Incorporation in Cadmium Oxalate Crystals Grown by Gel Method. Bulletin of Materials Science, 16, 365-370. http://dx.doi.org/10.1007/BF02759549

[7] Pactor, A. (1977) The Precipitation of Alkaline-Earth Metal Molybdate Powders from Aqueous Solution. Crystal Numbers, Final Morphology and Sizes. Kristall und Technik, 12, 729-735. http://dx.doi.org/10.1002/crat.19770120709

[8] Arora, S.K. and Abraham, T. (1977) Controlled Nucleation of Cadmium Oxalate in Silica Hydro Gel and Characterization of Grown Crystals. Journal of Crystal Growth, 52, 851-857. http://dx.doi.org/10.1016/0022-0248(81)90388-2

[9] Dennis, J. and Henish, H.K. (1967) Impurity Distribution in Single Crystals. III. Impurity Heterogeneities in Single Crystals. Journal of The Electrochemical Society, 114, 738-742. http://dx.doi.org/10.1149/1.2426720

[10] Diaz-Guemes, M.I., Bhatti, A.S. and Dollimere, D. (1986) Thethermal Decomposition of Oxalates. Part. 21. The Preparation and Thermal Decomposition of an Oxy Molybdenum (VI). Themochemica Acta, 106, 125-132.

http://dx.doi.org/10.1016/0040-6031(86)85123-1 
[11] Parekh, B., Vyas, P.M., Vasant, S.R. and Joshi, M.J. (2008) Thermal, FTIR and Dielectric Studies of Gel Grown $\mathrm{Na}_{2} \mathrm{C}_{2} \mathrm{O}_{4}$ Crystals. Bulletin of Materials Science, 31, 143-147. http://dx.doi.org/10.1007/s12034-008-0025-1

[12] Drago, R.S. (1965) Physical Methods in Inorganic Chemistry, 219.

[13] Dalal, P.V. (2013) Nucleation Controlled Growth of Cadmium Oxalate Crystals in Agar Gel and Their Characterization. Indian Journal of Materials Science, 7, 729-735. http://dx.doi.org/10.1155/2013/682950

[14] Selasteen, F.D. and Raj, S.A.C. (2016) Influences of Sodium in Cadmium Oxalate Dehydrate Single Crystals-Synthesis, Growth and Characterization. Iosrd International Journal of Physics, 2, 29-33. http://dx.doi.org/10.2202/ijphy.v2i1.95

[15] Raj, A.M.E. (2008) Optimized Growth and Characterization of Cadmium Oxalate Single Crystals in Silica Gel. Solid State Sciences, 10, 557-562. http://dx.doi.org/10.1016/j.solidstatesciences.2007.10.019

[16] Singh, O. and Singh, K. (2012) Thermal Behavior and Decomposition Kinetics of a Pr(III) with 4,6-Dihydroxyaurone. International Journal of Science and Nature, 3, 632-634.

[17] Saiyed, B.A. (2012) The Study of Thermal Stability and Decomposition in Cadmium Oxalate Single Crystals. IJRT, 1, 8.

[18] Raj, A.M.E., Jayanthi, D.D. and Jothy, V.B. (2009) Crystal Structure and Thermal Characterization of Cadmium Oxalate $\mathrm{CdC}_{2} \mathrm{O}_{4}$ and Barium Doped Cadmium Oxalate $\mathrm{Ba} \cdot \mathrm{Cd} \mathrm{C}_{2} \mathrm{O}_{4}$ Single Crystals Grown in Silica Gel. Inorganica Chemical Acta, 362, 1535-1540. http://dx.doi.org/10.1016/j.ica.2008.07.025

[19] Ashour, A., EI-Kadry, N. and Mahmoud, S.A. (1995) On the Electrical and Optical Properties of CdS Films Thermally Deposited by a Modified Source. Thin Solid Films, 269, 117-120. http://dx.doi.org/10.1016/0040-6090(95)06868-6

[20] Tauc, J., Grigorovici, R. and Vancu, A. (1966) Optical Properties and Electric Structure of Amorphous Dielectric Germanium. Physica Status Solidi B, 15, 627-637. http://dx.doi.org/10.1002/pssb.19660150224

[21] Arora, S.K. (1982) Indentation Study of Cadmium Oxalate Trihydrate Single Crystals. Journal of Materials Science, 17, 2825-2830. http://dx.doi.org/10.1007/BF00644657 